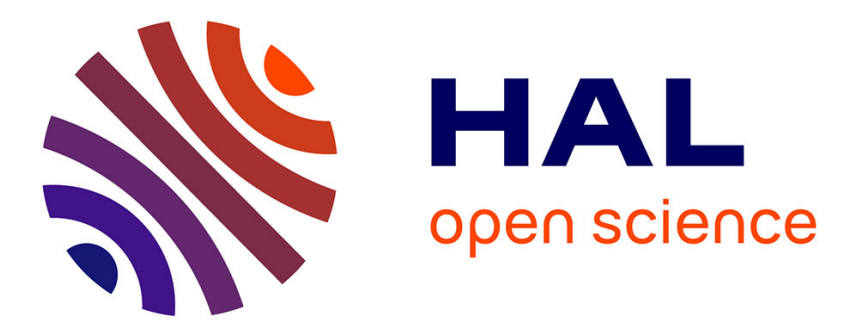

\title{
L'usage de cannabis chez les adolescents et les jeunes adultes : comparaison des consommations européennes
}

François G. E. Beck, Stéphane Legleye, Stanislas Spilka

\section{To cite this version:}

François G. E. Beck, Stéphane Legleye, Stanislas Spilka. L'usage de cannabis chez les adolescents et les jeunes adultes: comparaison des consommations européennes. Santé Publique, 2007, 13 (6), pp.481-488. halshs-00283011

\section{HAL Id: halshs-00283011 https://shs.hal.science/halshs-00283011}

Submitted on 29 May 2008

HAL is a multi-disciplinary open access archive for the deposit and dissemination of scientific research documents, whether they are published or not. The documents may come from teaching and research institutions in France or abroad, or from public or private research centers.
L'archive ouverte pluridisciplinaire HAL, est destinée au dépôt et à la diffusion de documents scientifiques de niveau recherche, publiés ou non, émanant des établissements d'enseignement et de recherche français ou étrangers, des laboratoires publics ou privés. 


\title{
L'usage de cannabis chez les adolescents et les jeunes adultes : comparaison des consommations européennes
}

\author{
The levels of use of cannabis by adolescents and young adults: \\ a comparison of European consumption
}

\author{
François Beck (1), (2), Stéphane Legleye (3), (4), Stanislas Spilka (3)
}

\begin{abstract}
Résumé : Les Français figurent parmi les premiers consommateurs de cannabis en Europe, que ce soit selon les enquêtes en population générale adulte des différents pays européens ou selon l'enquête Espad menée auprès de plus de cent mille adolescents de 15-16 ans dans la plupart des pays d'Europe. Parmi les nations présentant les politiques les plus répressives en matière d'usage de stupéfiants, le niveau de consommation du cannabis apparaît particulièrement élevé en France et au plus bas en Suède, alors qu'à l'opposé les Pays-Bas occupent une place médiane. Plus que la législation, ce sont davantage les réseaux de relation noués entre les jeunes qui déterminent les choix de consommation d'un produit comme le cannabis, dans la mesure où les pratiques d'un adolescent s'avèrent très liées aux représentations et aux usages de ses pairs.
\end{abstract}

Mots-clés : Cannabis - comparaisons européennes - enquêtes en population générale adolescents - addictions - drogues - cartographie.

\begin{abstract}
Summary: The French figure among the largest consumers of cannabis in Europe, both according to general population surveys of adults from various European countries and the European School Survey Project on Alcohol and Other Drugs (ESPAD survey) carried out among more than 100,000 15-16 year old adolescents from most countries in Europe. Among the countries surveyed who have the most strict and suppressive policies concerning the use of psychotropic drugs, the level of consumption of cannabis appears particularly high in France and lowest in Sweden; although in a country diametrically opposed in terms of policy measures in this regard, the Netherlands (a country which carries the image of much freedom and leeway in this matter), actually occupies the position of average consumption rates in the middle of the two extreme cases aforementioned. Even more important than legislation is the presence of networks of close-knit relationships between young people; this is a critical aspect which has a strong influence in determining the choice whether or not to consume a product such as cannabis. In this manner, adolescents' behaviour and practices are integrally linked to the opinions, perceptions and use of his/her peers.
\end{abstract}

Keywords: Cannabis - European comparative surveys - general population surveys adolescents - addictions - drugs - cartography.

(1) Responsable du Département Observation et analyse des comportements de santé. Institut National de Prévention et d’Éducation pour la Santé (INPES) - 42, boulevard de la Libération - 93203 Saint Denis Cedex. francois.beck@inpes.sante.fr

(2) CESAMES, Centre de recherche Psychotropes, Santé mentale, Société (CNRS UMR 8136, INSERM U611, Université René Descartes Paris V).

(3) Observatoire Français des Drogues et des Toxicomanies.

(4) Inserm U669, Université Paris XI. 


\section{Introduction}

Devant la généralisation de l'usage de cannabis observée en France et dans la plupart des pays européens depuis le début des années 1990, il apparaît particulièrement utile de faire le point sur les comparaisons internationales des niveaux d'usage en populations jeune adulte et adolescente [8]. Les efforts de l'Observatoire Européen des Drogues et Toxicomanies (OEDT), pour rendre les enquêtes en population générale des différents pays comparables grâce à des protocoles standardisés, permettent désormais de dresser une cartographie européenne des usages de cannabis au cours de l'année parmi les adultes ou les adolescents [17]. L'intérêt de ce travail comparatif est d'apporter des éléments d'évaluation à mettre en regard des stratégies de politique publique et de santé publique mises en œuvre dans les différents pays. En effet, si la littérature scientifique sur les différents facteurs associés à l'usage du cannabis apparaît très abondante, celle portant sur les comparaisons internationales se révèle beaucoup plus pauvre $[10,23]$ même si un regain d'intérêt récent sur cette question doit être signalé [12, 15, 22].

\section{Matériel et méthode}

Les données concernant les jeunes adultes proviennent des enquêtes nationales en population générale les plus récentes dans chaque pays, l'année de référence variant de 2000 à 2005 selon la disponibilité, et étant le plus souvent 2003. Si la plupart des pays peuvent fournir des résultats sur la tranche d'âge 15-34 ans, les données disponibles ne correspondent pas toujours exactement à cette tranche (pour le Danemark, la Pologne et le Royaume-Uni, elle est de 16 à 34 ans, pour la République tchèque, la Slovaquie, la Hongrie, la Suède et l'Allemagne, elle est de 18 à 34 ans ; pour la Suisse, il s'agit de la tranche 15-39 ans). La méthodologie de la plupart de ces enquêtes est décrite avec précision dans le bulletin statistique de l'OEDT, disponible en ligne ${ }^{(5)}$.

L'Eurobaromètre 2002 est, pour sa part, une enquête menée auprès des 15-24 ans dans les différents pays de l'Europe des quinze, suivant une méthodologie similaire (enquête réalisée avec enquêteur, en face à face au domicile des enquêtés sur un échantillon aléatoire mais de petite taille, variant de 190 à 550 selon le pays).

Pour les adolescents, l'enquête European School Survey Project on Alcohol and Other Drugs (ESPAD) est menée en milieu scolaire dans une trentaine de pays européens tous les quatre ans depuis 1995. Grâce à la dernière édition de 2003, il est possible d'observer les usages des jeunes adolescents scolarisés nés en 1987 (donc âgés de 15-16 ans au moment de l'enquête). L'enquête a été menée au même moment, selon une méthodologie standardisée, dans l'ensemble des pays participants. Les données ont été recueillies à l'aide d'un questionnaire standard auprès d'échantillons représentatifs à l'échelle nationale. Elles concernent un total d'environ 100000 élèves de collège et de lycée. Ce questionnaire auto-administré a été conçu pour être adapté à l'interrogation des adolescents sur un sujet sensible. La taille des échantillons varie selon les pays de 550 à 6000 élèves [13]. En

(5) http://stats06.emcdda.europa.eu/download/gpstab21.xls 
France, l'enquête s'est effectuée sous la responsabilité scientifique de l'OFDT et de l'équipe "santé de l'adolescent» de l'INSERM en partenariat avec le ministère de la Jeunesse, de l'Éducation Nationale et de la Recherche (MJENR).

Les indicateurs utilisés sont l'expérimentation (au moins un usage au cours de la vie), l'usage récent (au moins un usage au cours des douze derniers mois), l'usage actuel (au moins un usage au cours des trente derniers jours). Le terme usage sans précision désigne l'ensemble de ces pratiques.

\section{Résultats}

En Europe, les niveaux d'usage actuels (au cours des douze derniers mois) de cannabis des jeunes adultes varient de $3 \%$ en Grèce à $22 \%$ en République tchèque. Ces enquêtes permettent de constater que la France, la République tchèque, l'Espagne et le Royaume-Uni présentent les taux d'usage au cours de l'année de cannabis les plus élevés au sein des pays européens. Dans tous les pays européens, les hommes s'avèrent plus fréquemment usagers de cannabis que les femmes [17].

Depuis le début des années 1990, dans les différents pays européens où plusieurs enquêtes en population générale ont pu être répétées dans le temps, le niveau de l'usage actuel de cannabis des jeunes adultes a augmenté de façon assez nette en Allemagne, au Danemark, et dans une moindre mesure aux Pays-Bas, en Finlande et en Suède. Dans les pays où la prévalence était plus élevée au début de la décennie, tels que le Royaume-Uni et l'Espagne, la hausse s'est avérée moins marquée. En France, il semble que la diffusion du cannabis ait atteint en 2002 un pourcentage record en Europe pour l'usage actuel de cannabis, avant de retrouver en 2005 le niveau de l'année 2000 [5].

La cartographie proposée grâce au travail de réseau de l'OEDT peut être mise en regard de celle réalisée à partir des données de l'Eurobaromètre sur les usages au cours des trente derniers jours des 15-24 ans [11]. La logique s'y trouvait radicalement différente puisque les quinze enquêtes ont été montées au même moment avec une méthodologie identique, mais en contrepartie les échantillons étaient de petite taille (de 190 à 550 selon le

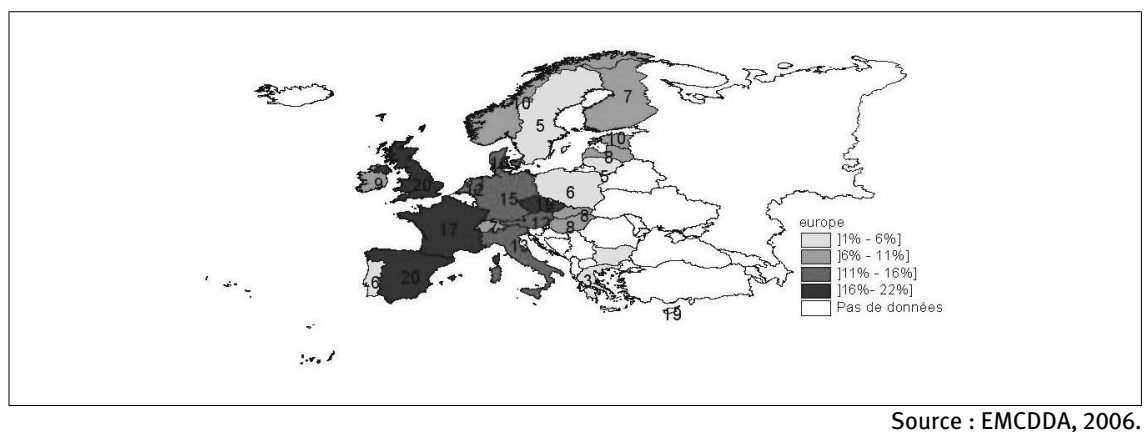

Carte 1 : L'usage de cannabis au cours de l'année selon les pays parmi les jeunes adultes. 
Tableau I : Usage de cannabis au cours de la vie et du mois parmi les 15-24 ans

\begin{tabular}{|l|c|c|}
\hline \multicolumn{1}{|c|}{ États membres } & Expérimentation (vie) \% & Usage au cours du mois \% \\
\hline Danemark $\mathrm{n}=454$ & $47,0[42,4-51,6]$ & $12,2[9,2-15,2]$ \\
\hline France $\mathrm{n}=447$ & $44,9[40,3-49,5]$ & $19,8[16,1-23,5]$ \\
\hline Royaume-Uni $\mathrm{n}=548$ & $37,0[33,0-41,0]$ & $13,4[10,6-16,2]$ \\
\hline Pays-Bas $\mathrm{n}=453$ & $35,3[30,9-39,7]$ & $12,2[9,2-15,2]$ \\
\hline Espagne $\mathrm{n}=450$ & $29,4[25,2-33,6]$ & $15,0[11,7-18,3]$ \\
\hline Luxembourg $\mathrm{n}=189$ & $27,3[21,1-33,5]$ & $4,5[1,4-7,6]$ \\
\hline Allemagne $\mathrm{a} \mathrm{n}=539$ & $25,6[21,6-29,6]$ & $8,3[5,8-10,8]$ \\
\hline Belgique $\mathrm{n}=456$ & $24,7[20,7-28,7]$ & $9,5[6,7-12,3]$ \\
\hline Irlande $\mathrm{n}=524$ & $24,2[20,2-28,2]$ & $8,7[5,9-11,5]$ \\
\hline Finlande $\mathrm{n}=402$ & $19,2[15,5-22,9]$ & $5,6[3,6-7,6]$ \\
\hline Autriche $\mathrm{n}=541$ & $17,9[14,6-21,4]$ & $4,6[3,7-6,5]$ \\
\hline Italie $\mathrm{n}=450$ & $17,2[13,9-20,7]$ & $7,8[5,2-10,4]$ \\
\hline Suède $\mathrm{n}=477$ & $16,5[13,1-19,6]$ & $3,9[2,3-5,5]$ \\
\hline Portugal $\mathrm{n}=457$ & $14,1[10,8-17,4]$ & $4,9[2,9-6,9]$ \\
\hline Grèce $\mathrm{n}=469$ & $4,8[2,8-6,8]$ & $1,3[0,3-2,3]$ \\
\hline
\end{tabular}

(a) Le niveau d'usage de cannabis apparaît plus élevé dans les anciens Länders (ex-RFA) que dans les nouveaux ( $27,2 \%$ vs $18,5 \%$ pour l'usage au cours de la vie et $8,8 \%$ vs $6,0 \%$ pour l'usage au cours du mois). Lecture : entre crochets figure l'intervalle de confiance à $95 \%$ de chacune des estimations fournies. Source : Eurobaromètre 2002.

pays). Les estimations fournies étaient donc imprécises et les méthodes d'enquête - rencontre des personnes en face-à-face au domicile des enquêtés - potentiellement inductrices de biais pour les questions portant sur l'usage de drogues.

Par rapport aux données collectées par l'OEDT auprès des différents points focaux européens, une certaine cohérence géographique apparaît, la Suède, la Grèce, la Finlande et le Portugal figurant selon les deux systèmes d'observation dans le groupe des pays peu consommateurs. Les intervalles de confiance (qui ont été recalculés ici mais ne figurent pas dans la publication de l'Eurobaromètre) donnent toutefois une idée de la faible précision des estimations de ces dernières données et permettent ici de nuancer les écarts observés.

La France se situe également en tête des pays européens pour les adolescents. La proportion d'élèves de 16 ans qui déclarent en avoir déjà consommé au moins une fois s'y trouve ainsi environ deux fois supérieure à la moyenne de l'ensemble des pays ( $38 \%$ contre $21 \%$ ). Le premier pays pour l'usage au cours de la vie (expérimentation) est la République tchèque avec un taux de $44 \%$, mais des taux élevés sont également rapportés en Irlande, au Royaume-Uni ou encore en Suisse (38-40\%). À l'opposé, des pays comme la Grèce, la Roumanie ou encore la Suède rapportent des taux particulièrement faibles (de $3 \%$ à $7 \%$ ). Les jeunes Français sont aussi parmi les premiers consommateurs de cannabis au cours du mois ( $22 \%$ vs $9 \%$ ). Ils figurent avec les Suisses, les Anglais et les Tchèques dans un groupe dont les niveaux sont compris entre $16 \%$ et $22 \%$, devançant largement ceux des autres européens. 


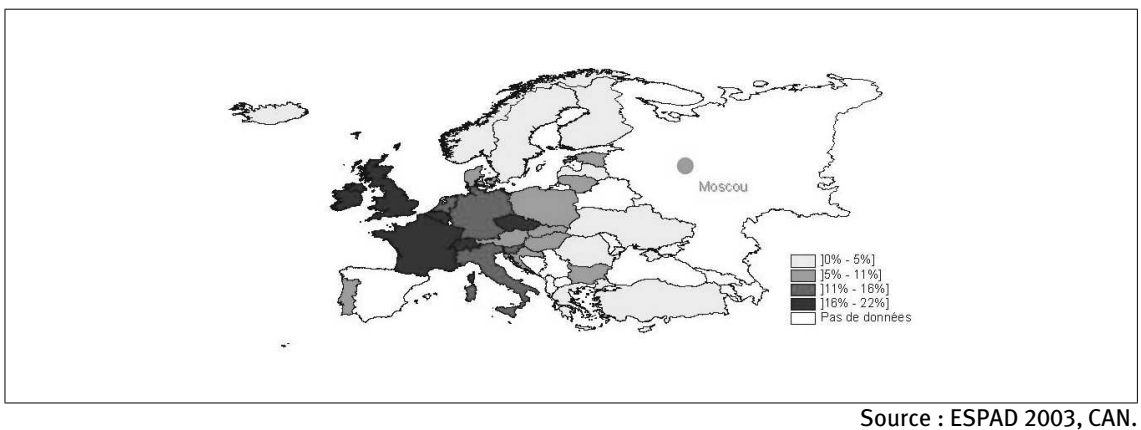

Carte 2 : L'usage de cannabis au cours du mois à 15-16 ans selon les pays.

La géographie européenne de l'usage de cannabis parmi les adolescents oppose, de façon encore plus nette que pour les jeunes adultes, l'Europe occidentale à l'Europe du nord et de l'est, les niveaux d'usage diminuant progressivement de la façade atlantique à Moscou. Parmi les pays d'Europe centrale pour lesquels la consommation au cours du mois s'avère relativement modérée, la République tchèque occupe, là encore, une place particulière avec un niveau d'usage déclaré comparable à celui de la France. $\mathrm{Si}$, comme en population adulte, les écarts entre les usages féminins et masculins sont généralement importants, il existe, en revanche, des pays où le sex-ratio est proche de 1 (l'Irlande, la Finlande, la Norvège et la Suède).

La répétition de l'enquête ESPAD à l'identique à quatre ans d'écart permet également de dégager des tendances montrant que le cannabis s'est diffusé de plus en plus massivement en Europe occidentale au cours des années 1990, avant de se stabiliser ces dernières années. Toutefois, les tendances à la hausse dans la plupart des pays d'Europe centrale et orientale, augurent peut-être d'une poursuite de la diffusion compte tenu des faibles niveaux observés. Entre 1999 et 2003, aucun pays ne présente de diminution significative des niveaux d'usage de cannabis. L'expérimentation progresse dans pratiquement un tiers des pays, essentiellement des pays d'Europe centrale et orientale (Bulgarie, République tchèque, Hongrie, Pologne...) mais aussi en Irlande et au Portugal. La consommation au cours du mois se révèle stable dans l'ensemble de l'Europe, seuls trois pays présentent des augmentations significatives de l'usage récent de cannabis: la Bulgarie, la Slovaquie mais aussi le Royaume-Uni où la hausse ( $20 \%$ vs $16 \%$ en 1999), intervient après une forte période de baisse entre 1995 et 1999 (16\% vS $24 \%$ en 1995). En France, l'usage de cannabis semble se stabiliser laissant penser qu'un palier a été atteint après une décennie durant laquelle la consommation de cannabis parmi les adolescents a progressé de manière importante comme dans la plupart des pays européens. L'exercice 2007 de l'enquête ESPAD permettra de répondre en partie à ces questions et notamment de faire un point sur l'évolution de l'usage dans les pays où il semble pour l'instant resté circonscrit à une petite minorité de la population (Grèce, Roumanie, Pologne). 


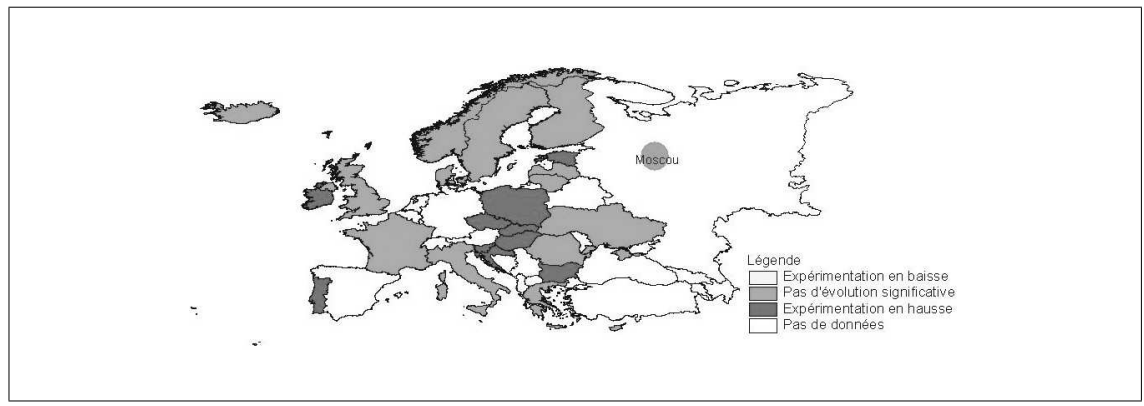

Carte 3 : Évolution 1999-2003 du niveau d'expérimentation de cannabis au cours du mois à 15-16 ans selon les pays.

\section{Discussion}

Les résultats présentés ici en population adolescente rejoignent en grande partie ceux observés grâce à l'enquête Health Behaviour in School-aged Children (HBSC) en 2002 [12], signe d'une certaine robustesse dans ces différentes observations. Si l'on peut désormais s'en réjouir, il convient de rappeler que cela n'a pas toujours été le cas : au début des années 1990, les données étaient plutôt présentées sous forme de tableaux utiles pour souligner les disparités de méthodes et invitant d'autant moins à des comparaisons simples qu'ils étaient assortis d'une litanie de précautions de lecture [20].

D’autres données peuvent également être mobilisées pour mettre en perspective ces comparaisons : par exemple, aux États-Unis, le niveau était de $21 \%$ en 2006 parmi les 18-34 ans [21], tandis qu'en Australie il était de $24 \%$ la même année [2]. La cartographie européenne de l'usage actuel de cannabis offre un visage contrasté où les pays d'Europe occidentale, et notamment la France, l'Angleterre et l'Espagne, présentent les niveaux les plus élevés (entre $17 \%$ et $22 \%$ ), tandis que les pays nordiques et d'Europe centrale affichent les plus faibles (compris entre $6 \%$ et $11 \%$ ). La République tchèque, avec un niveau d'usage de $19 \%$, se distingue toutefois nettement des autres pays de ce groupe.

Parmi les adolescents comme au sein de la population adulte, les écarts entre usages féminins et masculins s'avèrent généralement importants. Il existe toutefois des pays où cet écart est faible et parfois non significatif, comme par exemple les pays nordiques, ce rapprochement étant sans doute à mettre en regard d'une certaine émancipation féminine, comme cela a déjà pu être observé sur l'alcool $[3,9]$.

Au-delà de l'intérêt de mettre les pratiques en regard des différences culturelles, économiques et sociales, de telles comparaisons internationales peuvent jouer un rôle important dans la mise en regard des modèles de politique publique de gestion des drogues, des législations et de la mise en application des textes en vigueur. Une lecture rigoureuse de ces chiffres permet par exemple d'invalider les thèses soulignant les méfaits du laxisme des législations néerlandaises et espagnoles du début des années 1990. Parmi les pays d'Europe présentant les politiques les plus répressives en 
matière d'usage de stupéfiants, le niveau de consommation du cannabis apparaît élevé en France et au plus bas en Suède, alors qu'à l'opposé les Pays-Bas occupent une place médiane. Dans une récente étude s'appuyant aussi sur des données recueillies auprès d'échantillons représentatifs d'usagers réguliers de cannabis à Amsterdam et à San Francisco, a ainsi été montré que la décriminalisation n'entraînait pas de hausse des niveaux de consommation [19].

Il convient toutefois de nuancer ce propos et la portée générale des comparaisons européennes, dans la mesure où les populations ont, indépendamment du contexte législatif, des particularités socioéconomiques et culturelles susceptibles de peser sur leurs pratiques. La description du type de politique publique en vigueur dans un pays est une affaire complexe qui engage toutes les dimensions de l'intervention de l'État et de l'ensemble de la société sur les drogues [6]. De plus, les politiques des drogues en Europe apparaissent de plus en plus convergentes, orientées sur le versant sanitaire et fonctionnant de plus en plus par rapport à une unité de mesure commune: le risque [7]. Il est de ce fait souvent réducteur de qualifier la politique publique d'un pays de "répressive » ou de "tolérante ", et la lecture de ces comparaisons ne peut être qu'un élément dans la compréhension des différents systèmes et de leurs conséquences.

Cette lecture permet notamment de conforter l'idée que plus que la législation, ce sont davantage les réseaux de relation noués entre les jeunes qui déterminent les choix de consommation d'un produit comme le cannabis, dans la mesure où les pratiques d'un adolescent s'avèrent très liées aux usages de ses pairs $[14,16]$, en particulier ceux des amis les plus proches [4]. Plus qu'une incitation forcenée à la consommation, le groupe des pairs joue souvent un rôle important dans la structuration des représentations du cannabis et des risques liés à son expérimentation puis à son usage $[1,18]$. Notons enfin que les explications de l'usage par les pratiques des pairs ou par la législation ne sont pas concurrentes, mais s'avèrent plutôt complémentaires. La législation tente en effet d'agir sur le contexte, la disponibilité, les croyances à l'égard du cannabis et pèse de ce fait sur l'usage des pairs, qui lui-même apparaît fortement lié à des processus identitaires.

\section{REMERCIEMENTS}

L'enquête ESPAD est coordonnée par Bjorn Hibell et Barbro Andersson du Swedish Council for Information on Alcohol and Other Drugs (CAN) à Stockholm. Cette coordination est soutenue financièrement par le gouvernement suédois. Julian Vicente de l'European Monitoring Centre for Drugs and Drug Addiction (EMCDDA) assure la coordination du travail d'expertise mené au sein de l’Union européenne sur les enquêtes en population générale sur les usages de drogues. 


\section{BIBLIOGRAPHIE}

1. Aquatias S, Maillard I, Zorman M. Faut-il avoir peur du haschich ? Entre diabolisation et banalisation : les vrais dangers pour les jeunes. Paris, Syros 1999:224.

2. Australian Institute of Health and Welfare (AlHW) Statistics on Drug Use in Australia. Canberra: AlHW, 2006.

3. Beck F, De Peretti G, Legleye S. L'alcool donne-t-il un genre ? Travail, genre et sociétés 2006;15:141-60.

4. Beck F, Legleye S, Peretti-Watel P. Regards sur la fin de l'adolescence : consommation de produits psychoactifs dans l'enquête ESCAPAD 2000. Paris, OFDT 2000:220.

5. Beck F, Legleye S, Spilka S, Briffault X, Gautier A, Lamboy B, Leon C, Wilquin J-L. Les niveaux d'usage des drogues en France en 2005, exploitation des données du Baromètre santé 2005. Tendances 2006;48:6.

6. Beck F. Entre représentativité des échantillons et représentation des usages : l'apport des enquêtes en population générale à la compréhension des usages de drogues [Thèse de sociologie], Université René Descartes Paris V 2006:477.

7. Bergeron H. "Europeanisation of drug policies: from common principles to mutual agreement", in: Steffen M \& Lehto J (dir.). Europeanisation of health policies: Issues, challenges, innovations. Londres, Routeledge 2004:174-87.

8. Bless R, Korf D, Riper H, Diemel S. Improving the comparability of general population surveys on drug use in the European Union. Final report, commissioned by EMCDDA 1997:108.

9. Bloomfield K, Allamani A, Beck F, et al. Gender, culture and alcohol problems. A multi-national study, Charité Universitätsmedizin, Institute for Medical Informatics, Biometrics \& Epidemiology, Berlin, 2005.

10. Brook JS, Brook DW, Arenchibia-Mireles 0 , Richter L, Whiteman M. Risk factors for adolescent marijuana use across cultures and across time. J Genet Psychol 2001;162:357-74.

11. Callemien M. Les attitudes et opinions des jeunes citoyens de l'Union européenne par rapport aux drogues : Eurobaromètre 57.2, Rapport à la Direction Générale Justice et Affaires Intérieures de la Commission européenne, 2002:45 ; disponible sur : http://europa.eu.int/comm/justice_home/unit/drogue_fr.htm

12. Godeau E, Vignes C, Ter Bogt T, Nic Gabhainn S, Navarro F. Consommation de cannabis par les élèves de 15 ans. Données issued de l'enquête internationale HBSC/OMS dans 32 pays occidentaux. Alcoologie et addictologie 2006;28 (2):135-42.

13. Hibell B, Andersson B, Bjarnasson T, et al. The ESPAD Report 2003. Alcohol and Other Drug Use Among Students in 35 European Countries, Stockholm, The Swedish Council for Information on Alcohol and Other Drugs, CAN 2004:450.

14. Huba G, Bentler P. The role of peer and adult models for drug taking at different stages in adolescence. J Youth Adolesc 1980;9:449-65.

15. Kokkevi A, Nic Gabhainn S, Spyropoulou M and the Risk Behaviour Focus Group of the HBSC Early Initiation of Cannabis Use: A Cross-national European Perspective. Journal of Adolescent Health 2006;39 (5):712-9.

16. Miller $P$, Plant $M$. The family, peer influences and substance use: findings from a study of UK teenagers. Journal of Substance Use 2003;8:19-26.

17. Observatoire Européen des Drogues et Toxicomanies (OEDT) Annual report on the state of the drugs problem in the European Union and Norway. Lisbon: EMCDDA 2006:96.

18. Peretti-Watel P, Beck F, Legleye S. Les usages sociaux des drogues. PUF, Le lien social 2007:229.

19. Reinarman C, Cohen PDA, Kaal HL. The Limited Relevance of Drug Policy: Cannabis in Amsterdam and in San Francisco. American Journal of Public Health 2004;94:836-42.

20. Reuband KH. Drug use and drug policy in Western Europe: epidemiological findings in a comparative perspective. European Addiction Research 1995;1:32-41.

21. Substance Abuse and Mental Health Services Administration (SAMHSA) Summary of Findings from the 2006 National Household Survey on Drug Abuse. Rockville, MD: Office of Applied Studies 2007, table 1.12B.

22. Ter Bogt T, Schmid H, Nic Gabhainn S, Fotiou A, Vollebergh W. Economic and cultural correlates of cannabis use among mid-adolescents in 31 countries. Addiction 2006;101:241-51.

23. Zoccolillo M, Price R Jr, Tedd JI, Hwu HG. Antisocial personality disorder: comparisons of prevalence, symptoms, and correlates in four countries. In: Cohen P, Slomkowski C, Robbins LN, editors. Historical and geographical influences on psychopathology. Mahwah, NJ: Erlbaum 1999:249-78. 